\title{
Multi-scale modeling strategies in materials science-The quasicontinuum method
}

\author{
VIJAY B SHENOY \\ Materials Research Centre, Indian Institute of Science, Bangalore 560 012, India
}

\begin{abstract}
The problem of prediction of finite temperature properties of materials poses great computational challenges. The computational treatment of the multitude of length and time scales involved in determining macroscopic properties has been attempted by several workers with varying degrees of success. This paper will review the recently developed quasicontinuum method which is an attempt to bridge the length scales in a single seamless model with the aid of the finite element method. Attempts to generalize this method to finite temperatures will be outlined.
\end{abstract}

Keywords. Multi-scale models; quasicontinuum method; finite elements.

\section{Introduction}

Technological breakthroughs and experimental advances have made possible the manufacture of devices and direct observation of materials at the nanometer length-scale. These innovations have ushered a renewed interest in the theoretical understanding of the behaviour of materials with the goal of designing devices and materials starting from an atomic point of view. Properties of materials and devices are a result of phenomena at various lengthscales and require accurate modeling to capture the physics at each length-scale. For example, the plastic response of metals involves the core structure of individual dislocations, interactions of dislocations with each other and with grain boundaries. Thus the prediction of plastic behaviour requires modeling at different scales where input to a model at a higher scale will be the output form to the model at a lower scale (see figure 1). For example, the junction strength of dislocations which is required in dislocation dynamics simulations is obtained from an atomistic simulation. While well established modeling strategies exist for each length-scale, the thrust of research has shifted, in recent times, to developing modeling strategies that bridge the length-scales.

The quasicontinuum method pivots on a strategy which attempts to take advantage of both conventional atomistic simulations and continuum mechanics to develop a seamless methodology for the modeling of defects such as dislocations, grain boundaries and cracks, and their interactions. The key idea of the quasicontinuum method is the use of a full atomistic description of the material near the defect cores while a coarse grained finite element model is used far away from the defect cores and heterogeneity where the lattice is not highly distorted. The constitutive description of the material in the finite elements is also obtained from an atomistic calculation. This strategy drastically reduces the required computa- tional effort, and allows for the simulation of much larger systems than those possible in traditional atomistic models. At the present time, the methodology is fully developed for static calculations with interatomic potentials such as embedded atom method, at zero Kelvin-examples of such simulations include interaction of a grain boundary with external stresses, and the interaction of a lattice dislocation with a grain boundary. The future directions include extension of the method to the more sophisticated total energy descriptions such as density functional theory, and to finite temperature nonequilibrium simulations.

The paper is organized as follows: §2 deals with the quasicontinuum formulation for zero Kelvin statics, §3 contains formulation for dynamics at zero Kelvin; conclusions and future directions are summarized in $\$ 4$.

The quasicontinuum method was first formulated by Tadmor et al (1996) and reformulated by Shenoy et al (1998), Shenoy (1998) and Shenoy et al (1999). The present paper is an extract of Shenoy (1998).

\section{Statics at zero Kelvin}

The quasicontinuum method is constructed as an approximation theory to atomistics, i.e. a systematic construction that reduces to the exact atomistic theory when all the atomic degrees of freedom are considered. Also, the method is able to deal with cracks, grain boundaries and free surfaces in a uniform fashion. To this end, we start with the premise that the body is made of a large number of atoms, $N$. The presence of a crystalline reference configuration is exploited in the sense that for many regions of the crystal, it is unnecessary to save lists of atomic positions since they can be generated as needed by exploiting the crystalline reference state. A given atom in the reference configuration is specified by a triplet of inte- 
gers $\boldsymbol{l}=\left(l_{1}, l_{2}, l_{3}\right)$, and the grain to which it belongs. The position of the atom in the reference configuration is then given as,

$$
\boldsymbol{X}(\boldsymbol{l})=\sum_{a=1}^{3} l_{a} \boldsymbol{B}_{a}^{\mu}+\boldsymbol{R}^{\mu},
$$

where $\boldsymbol{B}_{a}^{\mu}$ is the $a$ th Bravais lattice vector associated with grain $G_{\mu}$ and $\boldsymbol{R}^{\mu}$ the position of a reference atom in grain, $G_{\mu}$ which serves as the origin for the atoms in grain $G_{\mu}$.
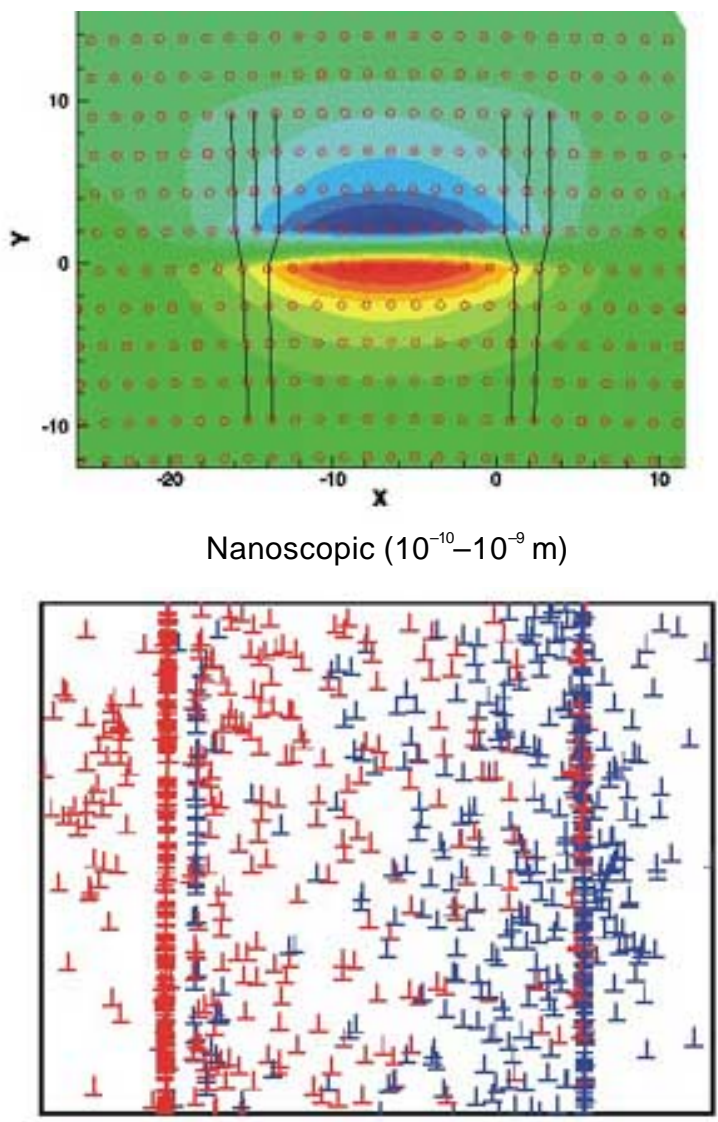

Mesoscopic $\left(10^{-8}-10^{-5} \mathrm{~m}\right)$

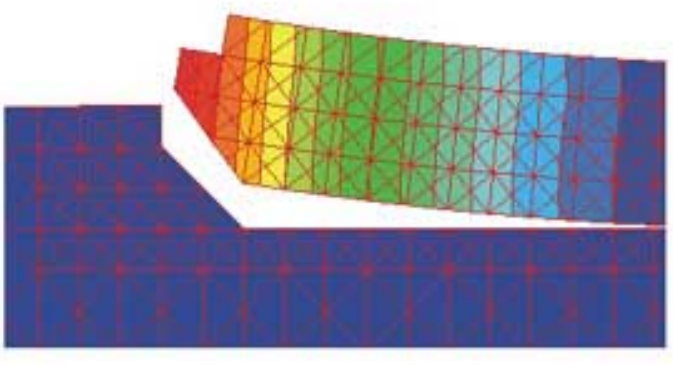

Macroscopic $\left(>10^{-4} \mathrm{~m}\right)$

Figure 1. Multiple scales in materials modeling.
Once the atomic positions have been given, from the standpoint of a strictly atomistic perspective, the total energy is given by the function

$$
E_{\text {tot }}=E_{\text {exact }}\left(\boldsymbol{x}_{1}, \boldsymbol{x}_{2}, \boldsymbol{x}_{3}, \ldots, \boldsymbol{x}_{N}\right)=E_{\text {exact }}\left(\left\{\boldsymbol{x}_{i}\right\}\right),
$$

where $\boldsymbol{x}_{i}$ is the deformed position of atom $i$. The following convention is adopted: capital letters refer to the undeformed configuration while lower case letters refer to the deformed configuration. The energy function in (2) depends explicitly upon each and every microscopic degree of freedom, and as it stands becomes intractable once the number of atoms exceeds one's current computational capacity. The problem of determining the minimum of the potential energy is, in the context noted above, nothing more than a statement of conventional lattice statics.

The first step in the construction of an approximation scheme is the selection of $R$ 'representative atoms' whose positions will be the only unconstrained degrees of freedom of the system. The position of any other atom in the system is obtained from a finite element mesh that is constructed with the representative atoms as nodes. Thus the approximate position $\boldsymbol{x}_{i}^{h}$ of any atom can be obtained by interpolation as

$$
\boldsymbol{x}_{i}^{h}=\sum_{\alpha} N_{\alpha}\left(\boldsymbol{X}_{i}\right) \boldsymbol{x}_{\alpha}
$$

where $N_{\alpha}\left(\boldsymbol{X}_{i}\right)$ is the finite element shape function centred around the representative atom, $\boldsymbol{\alpha}$ (which is also a FEM node), evaluated at the undeformed position, $\boldsymbol{X}_{i}$ of atom $i$. The finite element formulation therefore provides a complete kinematic description of the body on the specification of the representative atoms and construction of the mesh.

The next step in the process is to approximate the energetics, i.e. to develop an approximate energy function that depends only on the positions of the representative atoms. It is here that we make a very crucial assumption regarding the type of energy functionals that are dealt with. We assume that the atomistic formulation under consideration provides for a site-wise additive decomposition of the total energy

$$
E_{\mathrm{tot}}=\sum_{i=1}^{N} E_{i} .
$$

Such a decomposition is allowed in the embedded atom method and pair potential formulations but not in the case of more sophisticated quantum mechanics formulations such as density functional theory. While this assumption restricts the class of energy functionals that allow for the approximations discussed herein, it is believed that the method developed will nevertheless be useful in treating very large systems using the simpler atomistic formulations such as EAM and pair potentials that would otherwise have required the use of supercomputers. Now for 
the approximation scheme: notice that the sum in (4) runs over all the atoms in the body, and if it is to be computed using the approximate positions given by (3) it would still require the consideration of all atomic degrees of freedom, and therefore no reduction is achieved. It is here, then, that we make the crucial approximation to compute the total energy in that

$$
E_{\mathrm{tot}} \approx \sum_{\alpha=1}^{R} n_{\alpha} E_{\alpha}
$$

The crucial idea embodied in this equation surrounds the selection of some set of representative atoms, each of which, in addition to providing a kinematic description of the body, is intended to characterize the energetics of some spatial neighbourhood within the body as indicated by the weight, $n_{\alpha}$. As yet, the statement of the problem is incomplete in that summation weights, $n_{\alpha}$, have not yet been specified. We treat the problem of the determination of $n_{\alpha}$ in a manner analogous to determination of quadrature weights in the approximate computation of definite integrals. In the present context the goal is to approximate a finite sum ('definite integral' on the lattice) by an appropriately chosen quadrature rule where the quadrature points are the sites of the representative atoms. Physically, the quantity $n_{\alpha}$ may be interpreted as the 'number of atoms represented' by the representative atom, $\alpha$ The quadrature rule (5) is designed such that in the limit in which the finite element mesh is refined all the way down to the atomic scale (a limit that is denoted as fully refined) each and every atomistic degree of freedom is accounted for, and the quadrature weights are unity (each representative atom represents only itself). On the other hand, in the far field regions where the fields are slowly varying, the quadrature weights reflect the volume of space (which is now proportional to the number of atoms) that is associated with the representative atom, and this is where the continuum assumption is made i.e. in the existence of a well defined number density in space.

An additional energetic approximation in computing (5) that simplifies the energy calculations and also makes it possible to formulate boundary conditions which mimic those expected in an elastic continuum is now made. The essential idea is motivated by figure 2 , which depicts the immediate neighbourhood of a dislocation core. In particular, for this Lomer dislocation note the characteristic geometric signature of the core, viz. the pentagonal group of atoms in the core region. Consider the environments of two of the atoms in this figure, one (labeled $A$ ) in the immediate core region, and the other (labeled $B$ ) in the far field of the defect. It is evident that the environment of atom $A$ is nonuniform, and that each of the atoms in that neighbourhood experiences a distinctly different environment. By way of contrast, atom $B$ has an environment that may be thought of as emerging from a uniform deformation, and each of the atoms in its vicinity sees a nearly identical geometry.

As a result of these geometric insights, the energy, $E_{\alpha}$, may be computed from an atomistic perspective in two different ways, depending upon the nature of the atomic environment of the representative atom $\alpha$ Far from the defect core, the fact that the atomic environments are nearly uniform is exploited by making a local calculation of the energy in which it is assumed that the state of deformation is homogeneous and is well characterized by the local deformation gradient $\boldsymbol{F}$. To compute the total energy of such atoms, the Bravais lattice vectors of the deformed configuration, $\boldsymbol{b}_{a}$, are obtained from those in the reference configuration $\boldsymbol{B}_{a}$ via $\boldsymbol{b}_{a}=\boldsymbol{F} \boldsymbol{B}_{a}$. Once the Bravais lattice vectors are specified, this reduces the computation of the energy to a standard exercise in the practice of lattice statics.

On the other hand, in regions that suffer a state of deformation that is nonuniform, such as the core region around atom $A$ in figure 2, the energy is computed by building a crystallite that reflects the deformed neighbourhood from the interpolated displacement fields. The atomic positions of each and every atom are given exclusively by $\boldsymbol{x}=\boldsymbol{X}+\boldsymbol{u}(\boldsymbol{X})$, where the displacement field $\boldsymbol{u}$ is determined from finite element interpolation. This ensures that a fully nonlocal atomistic calculation is performed in regions of rapidly varying $\boldsymbol{F}$. An automatic criterion for determining whether to use the local or nonlocal rule to compute a representative atom's energy based on the variation of deformation gradient in its vicinity is presented by Shenoy et al (1999). The distinction between local and nonlocal environments has the unfortunate side effect of introducing small spurious forces referred to as 'ghost' forces at the interface between the local and nonlocal regions. A correction for this problem is also discussed in Shenoy et al (1999).

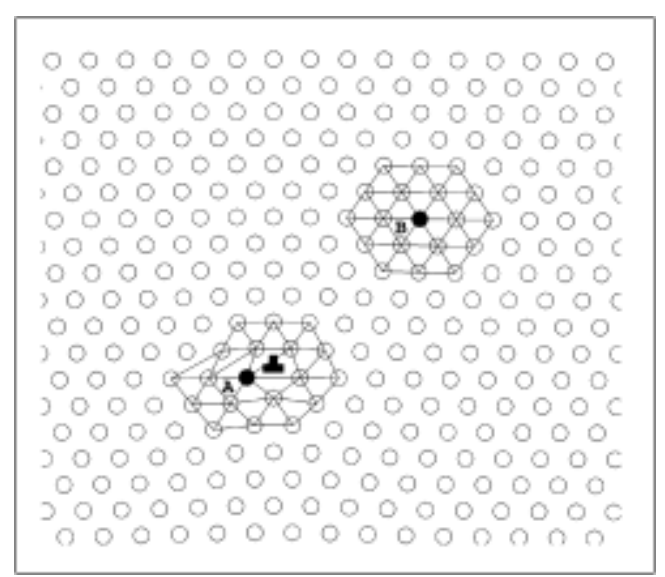

Figure 2. Atomic structure near the core of a Lomer dislocation in $\mathrm{Al}$. The atom, $A$, in the core region experiences an inhomogeneous environment while the environment of atom $B$ is nearly homogeneous. 
Once the total energy has been computed and both the kinematic and energetic bookkeeping have been settled, we are in a position to determine the energy minimizing displacement fields. As will be discussed below, there are a number of technical issues that surround the use of either conjugate gradient or Newton-Raphson techniques. Both of these techniques are predicted upon a knowledge of various derivatives of the total energy with respect to nodal displacements.

As noted in the introduction, one of the design criteria in the formulation of the method was that of having an adaptive capability that allowed for the targeting of particular regions for refinement in response to the emergence of rapidly varying displacement fields. For example, when simulating nanoindentation, the indentation process leads to the nucleation and subsequent propagation of dislocations into the bulk of the crystal. To capture the presence of the slip that is tied to these dislocations, it is necessary that the slip plane be refined all the way to the atomic scale. The adaption scheme allows for the natural emergence of such mesh refinement as an outcome of the deformation history. The adaption scheme, except for the adaption criterion, remains identical to that of Tadmor (1996).

We now list the essential points of the approximation scheme presented in this section:

(I) A subset of the total number of atoms that make up the body are selected (representative atoms) and their positions are treated as the only unknowns. The position of any other atom in the body is then obtained from a finite element mesh whose nodes correspond to the representative atoms.

(II) The energy of the system is also computed with the knowledge of energies of only the representative atoms. This is accomplished by the rule in (5).

(III) A further approximation in the computation of the energies of the representative atoms is made where the deformations are approximately homogeneous on the scale of the lattice.

\subsection{Application: dislocation-grain boundary interaction}

The interaction of dislocations with grain boundaries has been identified as an important factor governing the yield and hardening behaviour of solids. For example, the dependence of the yield stress on the grain size given by the celebrated Hall-Petch relationship, is explained using a pile-up model which assumes that dislocations are stopped by the grain boundary. In this section we illustrate how the QC method can be used to build realistic models that address the issue of the interaction of lattice dislocations with grain boundaries. For the specific grain boundary that we consider, we confirm the hypothesis that a pile-up will indeed occur, and that no-slip transmission takes place across the boundary.
We study the interaction of $\frac{a_{0}}{2}[\overline{1} 10]$ dislocations with a $\sum=21(2 \overline{4} \overline{1})$ symmetric tilt boundary in aluminum. Figure 3 shows a bicrystal, the top face (between $A$ and $B$ in figure 3) of which is subject to a kinematic boundary condition that mimics the effects of a rigid indenter. On attainment of a critical load, dislocations are nucleated at the point $A$, and they move towards the grain boundary. We investigate the nature of the interaction of these dislocations and the grain boundary through the consideration of the following questions: will the dislocation be absorbed by the boundary, and if so what is the result of this process? will the dislocation cause a sufficient stress concentration at the boundary so as to result in the nucleation of a dislocation in the neighbouring grain?

On application of the load, the bicrystal undergoes some initial elastic deformation and the first dislocation is nucleated when the displacement of the top face reaches $14.2 \AA$. This dislocation is driven into the boundary and is absorbed without an increase in the load level. Figures $4 \mathrm{a}, \mathrm{b}$ show the configuration of the grain boundary immediately before and after this nucleation event. It is seen that the dislocation absorption produces a step on the grain boundary. This process may be understood based on the DSC lattice by decomposing the Burgers vector into DSC lattice vectors (King and Smith 1980). In the present case, we find that

$$
\frac{a_{0}}{2}[\overline{1}, 1,0]=\frac{a_{0}}{14}[\overline{3} \overline{1} \overline{2}]+\frac{a_{0}}{7}[\overline{2} 41],
$$

where $\frac{a_{0}}{14}[\overline{3} \overline{1} \overline{2}]$ is the Burgers vector of a grain boundary dislocation parallel to the boundary and $\frac{a_{0}}{7}[\overline{3} 41]$ is perpendicular to the boundary plane. A careful examination of figure $4 \mathrm{~b}$ reveals that $\frac{a_{0}}{14}[\overline{3} \overline{1} \overline{2}]$ travels along the boundary, and stops on reaching the end of the nonlinear zone. On subsequent loading, another pair of Shockley partials are nucleated when the displacement of the rigid

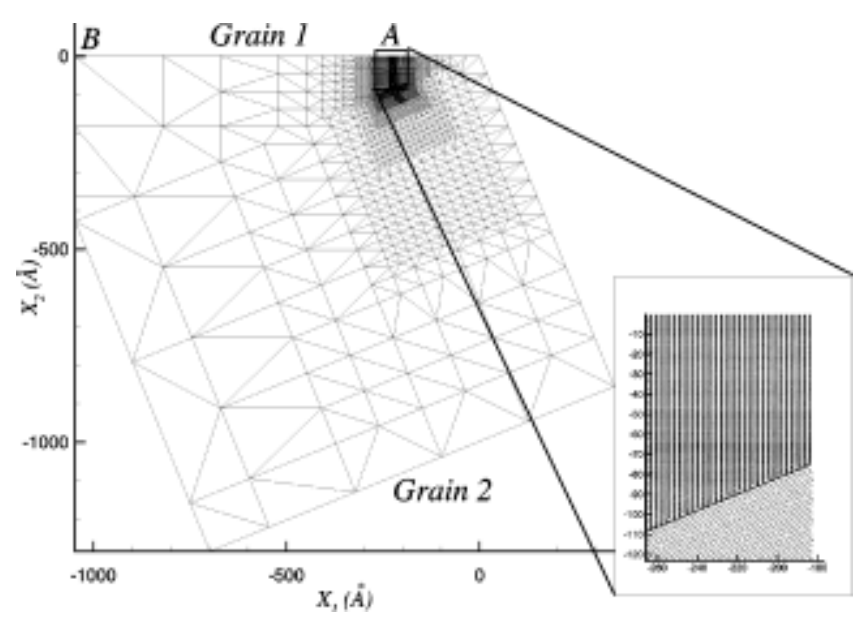

Figure 3. Mesh designed to model the interaction of dislocations and a grain boundary. Dislocations are generated at the point $A$ by rigidly indenting on the face $A B$ of the crystal. 


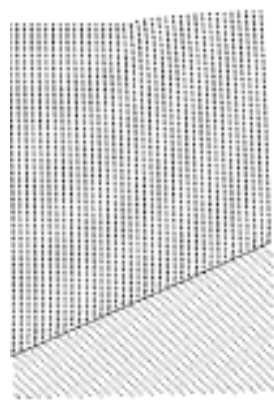

(a)

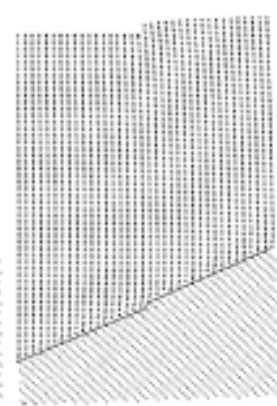

(b)

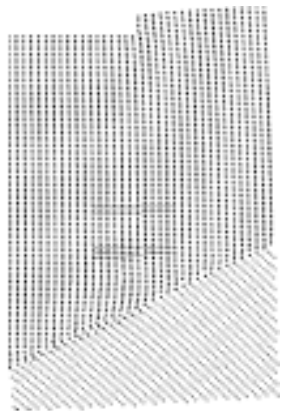

(c)
Figure 4. Snapshots of atomic configurations depicting the interaction of dislocations with a grain boundary. (a) Atomic configuration immediately before the nucleation of the partials, (b) atomic configuration immediately after the nucleation of the first set of partials which have been absorbed into the boundary and (c) the second pair of nucleated partials form a pile up.

indenter is $18.2 \AA$, which again does not result in any significant reduction of the load. Unlike the first pair, these dislocations are not immediately absorbed by the boundary, and they form a pile-up ahead of the boundary as shown in figure 4c. On additional indentation, these dislocations are also absorbed by the boundary. The simulation was terminated at this stage.

The neighbouring crystal shows no significant dislocation activity and thus it may be concluded that slip is not transmitted into the neighbouring grain across the boundary. The absorption of the dislocation resulted in a sliding motion of the grain boundary by the passage of a grain boundary dislocation and the formation of a step on the grain boundary. The formation of the step appears to result in the increased resistance of the boundary to dislocations, as is clear from the fact that a significantly higher stress level had to be attained before the absorption of the second dislocation. The type of dislocation grain boundary interaction seen here is the third type as classified by Shen et al (1988).

It is worth noting the significant computational saving obtained by the use of the QC method for this problem. The number of degrees of freedom used in the QC model was about $10^{4}$ while a complete atomistic model of this problem would have required more than $10^{7}$ degrees of freedom. The QC simulation required about $140 \mathrm{~h}$ on a DEC-Alpha work-station while a purely atomistic model would have required a parallel supercomputer.

\section{Dynamics at 0 kelvin}

The basic idea of the static quasicontinuum method presented in $\$ 2$ is carried forward to dynamics, in that representative atoms are chosen, and a finite element mesh is constructed with these as nodes. The kinematics of displacements and velocities are obtained by interpolation. The total energy (or Hamiltonian) of the system is also approximated in a similar fashion as in the case of statics.
To achieve this we start with an expression for the Hamiltonian (in a classical setting) as

$$
\begin{aligned}
H\left(\boldsymbol{x}_{1}, \ldots, \boldsymbol{x}_{N}, \boldsymbol{p}_{1}, \ldots, \boldsymbol{p}_{N}\right) & =\underbrace{E_{\mathrm{tot}}\left(\boldsymbol{x}_{1}, \ldots, \boldsymbol{x}_{N}\right)}_{\text {potentialenergy }}+\underbrace{\sum_{i} \frac{\boldsymbol{p}_{i}^{2}}{2 m}}_{\text {kineticenergy }} \\
& -\underbrace{\sum_{i} \boldsymbol{f}_{i} \cdot \boldsymbol{x}_{i}}_{\text {potential of external sources }},
\end{aligned}
$$

where the $\boldsymbol{x}_{i}$ is the position of the atom $i, \boldsymbol{p}_{i}$ its momentum, and $N$ the total number of atoms. The potential energy, $E_{\mathrm{tot}}$, is obtained from an atomistic formulation. It is assumed that all the atoms that make up the solid are the same species and the mass of each of them is assumed to be $m$. The evolution of this atomic system is governed by Hamilton's equations of motion (Goldstein 1980):

$$
\begin{aligned}
& \frac{\partial \boldsymbol{p}_{i}}{\partial t}=-\frac{\partial H}{\partial \boldsymbol{x}_{i}}=-\frac{\partial E_{\mathrm{tot}}}{\partial \boldsymbol{x}_{i}}+\boldsymbol{f}_{i}, \\
& \frac{\partial \boldsymbol{x}_{i}}{\partial t}=\frac{\partial H}{\partial \boldsymbol{p}_{i}}=\frac{\boldsymbol{p}_{i}}{m} .
\end{aligned}
$$

These equations have been widely used in molecular dynamics simulations such as in the work of Abraham et al (1997) which involves billion-atom studies of fast fracture in solids.

\subsection{Approximate Hamiltonian}

The goal of the dynamic quasicontinuum method is to develop an approximate Hamiltonian and derive evolution equations for the reduced degrees of freedom from the approximate Hamiltonian. As always, the first step is the selection of $R$ representative atoms whose positions and momenta are the reduced degrees of freedom. The position and velocity of any other atom may now be obtained using a finite element interpolation from the mesh constructed with these representative atoms as nodesthis provides a complete kinematic description of the dynamically deforming solid. As in the case of statics, constant strain triangles are used for this discretization procedure. It is here that the important assumption of 0 Kelvin temperature is made-the fields are assumed to be smooth enough to allow for a finite element interpolation. This will not be true at finite temperatures where thermal fluctuations result in these quantities varying wildly from one atomic site to the next.

Having obtained an approximate description of the kinematics, attention is now focussed on constructing an approximate Hamiltonian that depends only on the reduced degrees of freedom. Since the potential energy is assumed to possess the property of sitewise decomposability, one can put down the approximate Hamiltonian as 


$$
\begin{aligned}
H_{h}\left(\boldsymbol{x}_{1}, \ldots, \boldsymbol{x}_{R}, \boldsymbol{p}_{1}, \ldots, \boldsymbol{p}_{R}\right) & =\sum_{\alpha=1}^{R} n_{\alpha} E_{\alpha}\left(\boldsymbol{x}_{1}, \ldots, \boldsymbol{x}_{R}\right) \\
& -\sum_{\alpha=1}^{R} n_{\alpha} \overline{\boldsymbol{f}}_{\alpha} \cdot \boldsymbol{x}_{i}+\sum_{\alpha=1}^{R} n_{\alpha} \frac{\boldsymbol{p}_{\alpha}^{2}}{2 m},
\end{aligned}
$$

where $\alpha$ is a subscript over all representative atoms. The main point of (9) is that the kinetic energy term is approximated in exactly the same manner as the potential energy term. Also, from a finite element point of view, this is the lumped mass approach to dynamic problems. In the lumped mass approach the mass matrix for each element is taken to be a diagonal matrix, as opposed to the consistent mass matrix which is derived from the weak form of the equations of motion and is not diagonal (Hughes 1987). The lumped mass approach in conjunction with an explicit integration scheme provides a very efficient method to tackle dynamics problems.

The equations of motion will now be derived from the approximate Hamiltonian. Before we do that we will recast the expression in (9) such that it actually represents a Hamiltonian - the momenta and positions in a Hamiltonian must be canonically conjugate (see Goldstein 1980). To this end define the lumped mass, $M_{\alpha}$, of the representative atom $\alpha$ as

$$
M_{\alpha}=n_{\alpha} m,
$$

and define the lumped momentum, $\boldsymbol{P}_{\alpha}$ and lumped force, $\boldsymbol{F}_{\alpha}$ of the representative atom $\boldsymbol{\alpha}$ as

$$
\begin{gathered}
\boldsymbol{P}_{\alpha}=n_{\alpha} \boldsymbol{p}_{\alpha}, \\
\boldsymbol{F}_{\alpha}=n_{\alpha} \overline{\boldsymbol{f}}_{\alpha} .
\end{gathered}
$$

Using the definitions in (10) and (11) the expression in (9) can be rewritten as

$$
\begin{aligned}
H_{h}\left(\boldsymbol{x}_{1}, \ldots, \boldsymbol{x}_{R}, \boldsymbol{P}_{1}, \ldots, \boldsymbol{P}_{R}\right) & =E_{\mathrm{tot}}^{h}\left(\boldsymbol{x}_{1}, \ldots, \boldsymbol{x}_{R}\right) \\
& -\sum_{\alpha=1}^{R} \boldsymbol{F}_{\alpha} \cdot \boldsymbol{x}_{\alpha}+\sum_{\alpha=1}^{R} \frac{\boldsymbol{P}_{\alpha}^{2}}{2 M_{\alpha}},
\end{aligned}
$$

where the abbreviation

$$
E_{\mathrm{tot}}^{h}\left(\boldsymbol{x}_{1}, \ldots, \boldsymbol{x}_{R}\right)=\sum_{\alpha} n_{\alpha} E_{\alpha}\left(\boldsymbol{x}_{1}, \ldots, \boldsymbol{x}_{R}\right),
$$

is used. It is now evident from the form of (12) that $\boldsymbol{P}_{\alpha}$ is canonically conjugate to $\boldsymbol{x}_{\alpha}$ and that the expression truly represents a Hamiltonian system.

The equations of motion for the representative atom may now be derived using the Hamilton's equations as

$$
\begin{aligned}
\frac{\partial \boldsymbol{P}_{\alpha}}{\partial t} & =-\frac{\partial E_{\mathrm{tot}}^{h}}{\partial \boldsymbol{x}_{\alpha}}+\boldsymbol{F}_{\alpha}, \\
\frac{\partial \boldsymbol{x}_{\alpha}}{\partial t} & =\frac{\boldsymbol{P}_{\alpha}}{M_{\alpha}} .
\end{aligned}
$$

Using the definitions in (10) and (11) the above equations can be simplified as

$$
\begin{aligned}
\frac{\partial p_{\alpha}}{\partial t} & =-\frac{1}{n_{\alpha}} \frac{\partial E_{\mathrm{tot}}^{h}}{\partial \boldsymbol{x}_{\alpha}}+\overline{\boldsymbol{f}}_{\alpha}, \\
\frac{\partial \boldsymbol{x}_{\alpha}}{\partial t} & =\frac{\boldsymbol{p}_{\alpha}}{m},
\end{aligned}
$$

and it is this set of equations that is integrated to obtain the approximate evolution of the atomic system. As in the case of statics, representative atoms are classified as nonlocal and local-nonlocal representative atoms are treated using an exact atomistic rule, while the local representative atoms are treated using the homogeneous deformation gradients in the elements that surround the representative atom. The problem of ghost forces that accompanies this classification is not treated in the present case.

One of the advantages of the present formulation is the fact that if all atoms in the model are selected and treated in a nonlocal fashion, the results of the conventional molecular dynamics are recovered.

\subsection{Subcycling}

We shall now discuss the integration of the equations of motion (15). The standard method would be to use the central difference rule (Hughes 1987)

$$
\begin{aligned}
& \boldsymbol{x}^{n+1}=\boldsymbol{x}^{n}+\Delta t \boldsymbol{v}^{n}+\frac{\Delta t^{2}}{2} \boldsymbol{a}^{n}, \\
& \boldsymbol{v}^{n+1}=v^{n}+\frac{\Delta t}{2}\left(\boldsymbol{a}^{n}+\boldsymbol{a}^{n+1}\right),
\end{aligned}
$$

where $\boldsymbol{x}, \boldsymbol{v}$ and $\boldsymbol{a}$ represent the positions, velocities and accelerations of all the representative atoms. The superscripts denote the time step, and $\Delta t$ represents the step size. The step size, $\Delta t$, in the standard central difference scheme is taken to be equal for all the atoms. The central difference scheme is only conditionally stable; i.e. $\Delta t$ cannot exceed a critical value for the algorithm to be stable. In the case of a nonuniform mesh where some of the nodes are at atomic resolution the value of $\Delta t$ will be determined by the time step taken required for the stable integration of these fully refined atoms. This value is of the order of $10^{-15}-10^{-14} \mathrm{~s}$. All the atoms in the model have to be integrated with this time resolution.

Belytschko and coworkers (Belytschko et al 1979; Belytschko and Smolinski 1985; Belytschko and Lu 1993) have developed a method called subcycling where each node in the finite element has its own step size and each element information is updated only on its own time step. Their motivation was to treat dynamic problems in nonlinear/inelastic analysis so that savings in computational times can be substantial in that the constitutive equation need not be integrated at every time step for 
each element. Each element step size is computed by solving the eigenvalue problem

$$
\left|\boldsymbol{K}_{\mathrm{e}}-\omega_{\mathrm{e}}^{2} \boldsymbol{M}_{\mathrm{e}}\right|=0
$$

where $\boldsymbol{K}_{\mathrm{e}}$ is the element stiffness matrix, $\boldsymbol{M}_{\mathrm{e}}$ the lumped mass matrix and $\omega$ the frequency of vibration. Now the step size for the element, e, is given by

$$
\Delta t_{\mathrm{e}} \leq \frac{2}{\omega_{\mathrm{e}}^{\max }},
$$

where $\omega_{\mathrm{e}}^{\max }$ is the maximum eigenvalue obtained from (17). The nodal step size for each node is defined as the minimum step size of all the elements of which the given node is a part.

The smallest step size over all elements is taken as the step size, $\Delta t$, for the problem-this is assumed to be the basic unit of time.

$$
\Delta t=\min _{\mathrm{e}} \Delta t_{\mathrm{e}}
$$

Each element is then assigned an integer, $s_{\mathrm{e}}$, that corresponds to the number of units of this step that makes up the time step for this element, i.e.

$$
s_{\mathrm{e}}=\left[\frac{\Delta t_{\mathrm{e}}}{\Delta t}\right],
$$

which represents the number of steps of $\Delta t$ that are taken before the stresses and other internal variables in this element are updated. Similarly, each node is also assigned an integral number, $s_{\alpha}$, which is taken as the minimum value of $s_{\mathrm{e}}$ over the elements that touch node $\alpha$ The acceleration of the node $\alpha$ is updated only for every $s_{\alpha}$ steps of $\Delta t$. The scheme for integration of any node whose time step is $s_{\alpha}$ steps of the unit time is as follows

$$
\begin{aligned}
& \boldsymbol{x}_{\alpha}^{n+i}=\boldsymbol{x}_{\alpha}^{n+(i-1)}+\Delta t v_{\alpha}^{n+(i-1)}+\frac{\Delta t^{2}}{2} \boldsymbol{a}_{\alpha}^{n+(i-1)}, \\
& v_{\alpha}^{n+i}=v_{\alpha}^{n+(i-1)}+\Delta t \boldsymbol{a}_{\alpha}^{n+(i-1)}, \\
& \boldsymbol{a}_{\alpha}^{n+i}=\boldsymbol{a}_{\alpha}^{n+(i-1)} \quad\left(\forall i<s_{\alpha}\right) .
\end{aligned}
$$

Put in words (21) means this-assume that at the time step $n$ measured with the unit step that the acceleration of the node $\alpha$ was updated. Then for all the $s_{\alpha}$ steps from this step, the above algorithm is used to integrate the equation of motion for the node $\alpha$ and the acceleration of the representative atom $\alpha$ is updated at the time step $n+s_{\alpha}$.

The steps involved in the subcycling algorithm can now be enumerated as follows. First, all the element and nodal step sizes are computed. At every time step each element is visited, and if its time step is reached, the stresses in that element are updated. Each node is then visited and equations of motion are integrated based on (21) and the accelerations are computed if the nodal time step is reached. We should point out here that subcycling is effective for local representative atoms only as all nonlocal atoms live in fully refined regions and therefore automatically have the unit step size for their integration. Therefore, computational gains will be substantial only in cases where the bulk of the computation comes from local atoms at each time step.

The effective use of this theory is possible only when appropriate adaptive remeshing schemes are implemented. This would involve developing a more general criterion than that given in Shenoy et al (1999), which preserves the energy of the model when the mesh is adapted. No such scheme is developed here, and is certainly an important step forward for this method to be effective. The present strategy has been to adapt the mesh to the fully refined state a priori where the defects are expected. The representative atoms in these fully refined regions are initially treated using the local rule and subsequently turn nonlocal when approached by defectsthis is guaranteed by periodic updates in the status of the representative atoms during the integration process.

\subsection{Applications-Dynamic nanoindentation}

Tadmor (1996) studied the problem of quasistatic nanoindentation in aluminum using the quasicontinuum method. Not only was he able to compute the load displacement curves, he was also able to find the different structures that evolved when indentation was carried out on different crystal faces. In the present context, it is interesting to use the same geometry to explore the dynamic effects that might appear in nanoindentation.

The material in question is aluminum which is simulated using the embedded atom potentials developed by Ercolessi and Adams (1993). A single crystal aluminum block is subjected to the action of a rectangular rigid

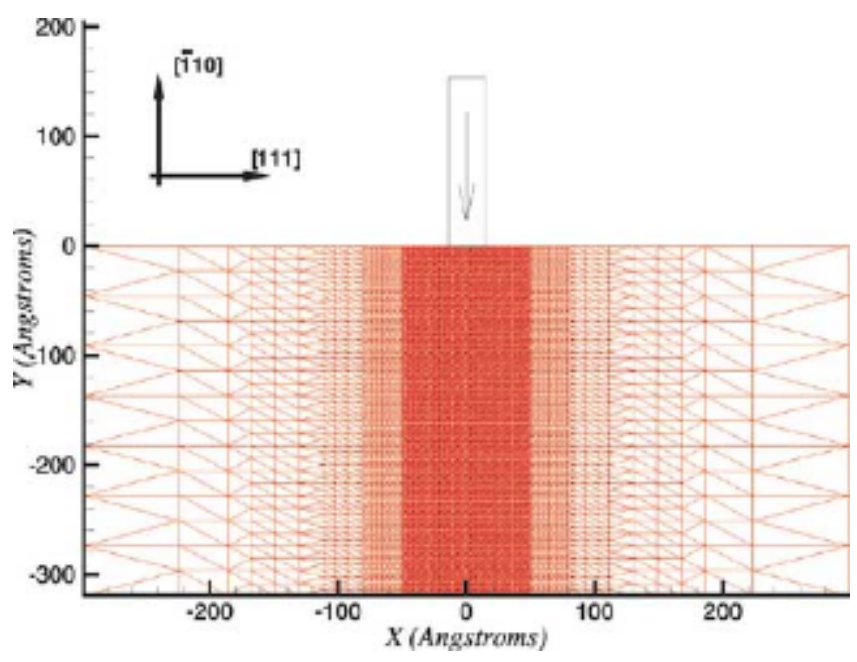

Figure 5. Mesh used for dynamic nanoindentation. 
Time $=0.6 \mathrm{ps}$
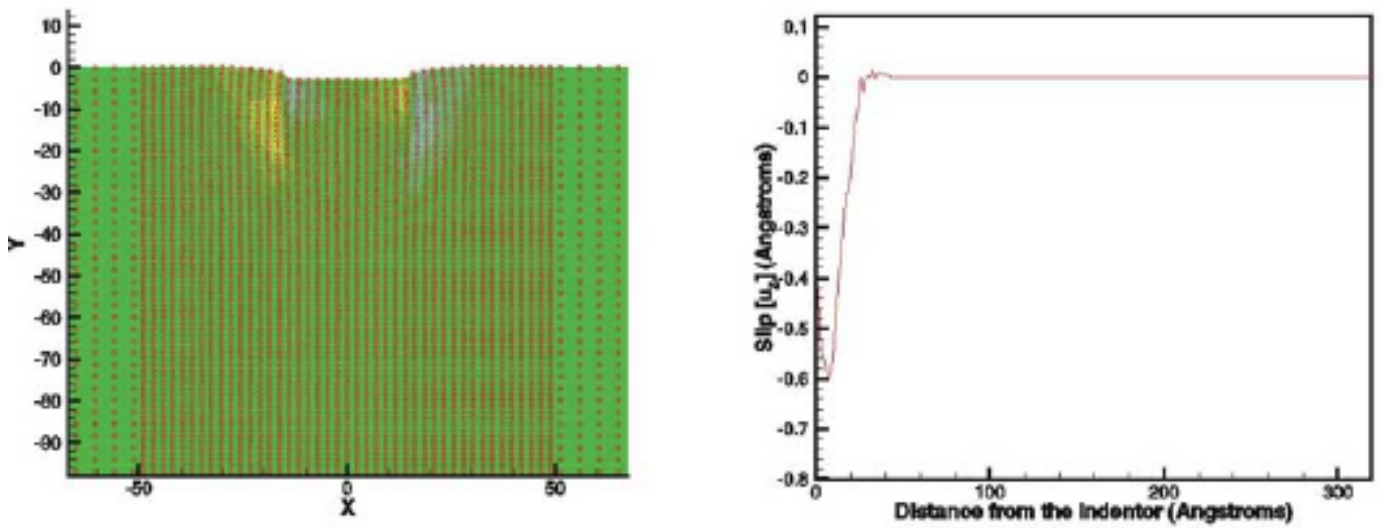

$\mathrm{TIme}=1.2 \mathrm{ps}$
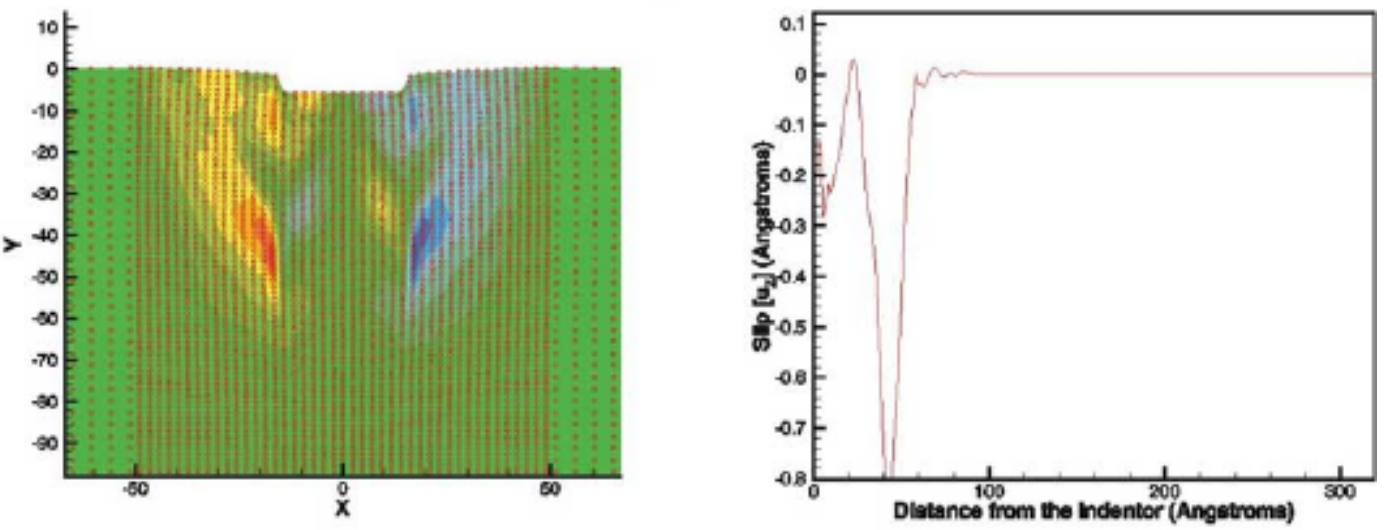

Time $=1.8 \mathrm{ps}$
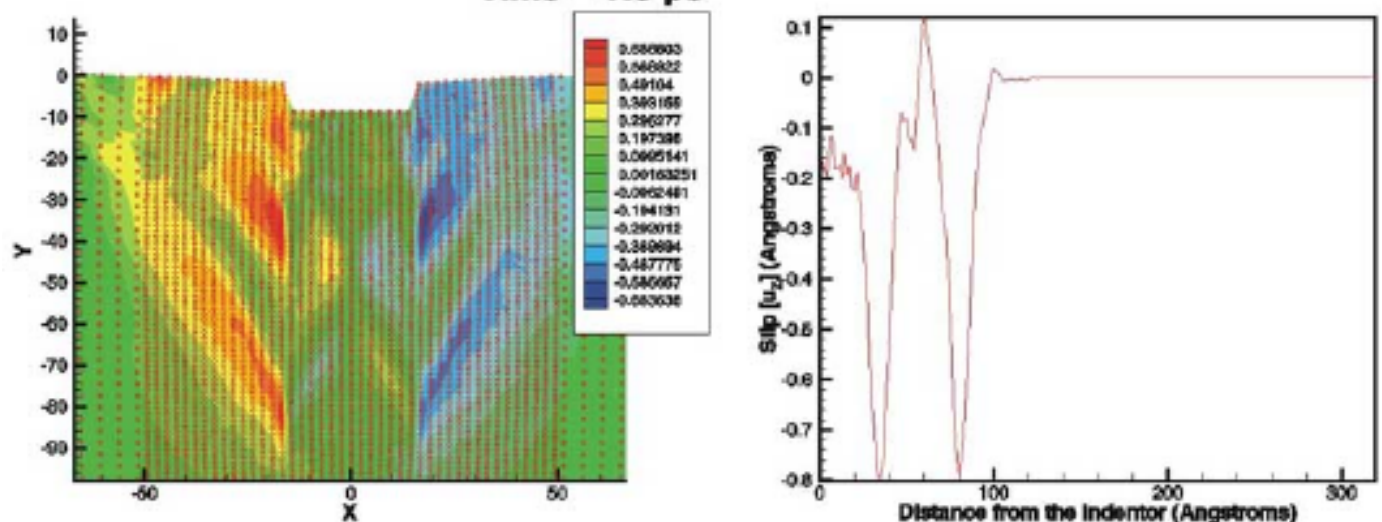

Figure 6. Left: Contour plot of the out of plane displacement, $u_{z}$; the numbers in the legend are in $\AA$. Right: Plot of jump in the out of plane displacement.

indenter whose width is $30 \AA$. The indenter pushes on a (110) face of the crystal-an orientation that is best suited to nucleate $\frac{1}{2}[\overline{1} 10]$ type dislocations. These dislocations would then run into the crystal. The indentation speed is chosen to be $5.0 \AA / \mathrm{ps}$ - seven percent of the longitudinal wave speed. The mesh used for the simulation is shown in figure 5. Since adaptive remeshing schemes are not used in the present set up, the mesh is refined to the atomic scale all along the slip plane where the dislocations are expected.

Figure 6 shows a contour plot of the out of plane displacement, $u_{z}$ and a plot of the jump in the displacement $\left[u_{z}\right]$ as a function of the distance from the indenter. These plots are of significance for the following reason. The $\frac{1}{2}[\overline{1} 10]$ dislocations in aluminum split into Shockley partials each of which have a screw character as well. 

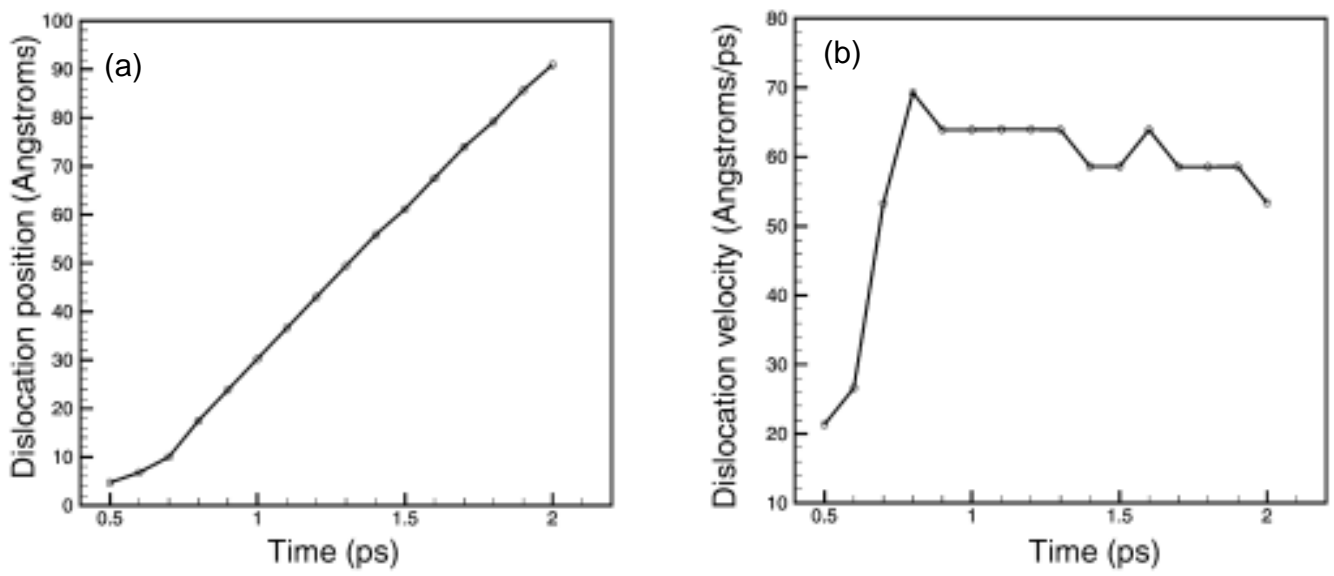

Figure 7. (a) Position of the lead set of dislocations measured from the free surface of the crystal as a function of time and (b) velocity of the lead set of dislocations as a function of time.

This shows up as an out of plane displacement in the simulations and therefore acts as the signature of the presence of dislocations. A careful study of figure 6 shows that the first set of dislocations is fully formed at about $0.6 \mathrm{ps}$ after the beginning of indentation and this set rushes into the crystal under the influence of the stress from the indenter. Subsequently, another set of dislocations is nucleated at $1.1 \mathrm{ps}$ and a third pair at $1.7 \mathrm{ps}$ (snapshots immediately after these events are shown in figure 6). All of these dislocations travel into the bulk of the crystal in procession.

One may calculate the speed of the dislocations as they rush through the crystal. The position of the dislocation is taken to be the distance at which the magnitude of the jump in the out of plane displacement is maximum. The jump in the displacement is plotted on the right hand column of figure 6. A plot of the position of the leading dislocation is shown in figure 7(a). The velocity of the dislocation can be computed using a central difference scheme from the data shown in figure $7(\mathrm{a})$ and this is shown in figure 7(b). It is seen from figure 7(b) that the dislocation rapidly accelerates to a velocity of about $70 \cdot 0 \AA / \mathrm{ps}$ and then later reaches an approximately constant velocity of about $61.0 \AA / \mathrm{ps}$, although it is clearly decelerating as it runs through the crystal. The behaviour of the other dislocations that are nucleated is also similar. The main conclusion of this study is that the dislocations are supersonic. A more detailed analysis of the results can be found in Shenoy (1998).

\section{Conclusions and future directions}

This paper presents a review of the quasicontinuum methodology. The quasicontinuum method is an approximation scheme for atomistics. The method is fully developed for the case of 0 Kelvin simulations.
Future work may be carried forward in two main directions. First, the method as it stands is applicable only to atomistic formulations which allow for a sitewise additive decomposition of the total energy. Thus, the method in its present form will not be applicable to density functional approaches where a thinning of the degrees of freedom will prove to be of great advantage. The second, and perhaps more challenging, direction is to extend this method to finite temperature simulations. For equilibrium simulations, a temperature dependent free energy is defined (Shenoy 1998) and minimization is carried out in a similar fashion as the 0 Kelvin case. For nonequilibrium systems, the exact governing principles of the system are not clear. An attempt has been made by Shenoy (1998) to develop a self-consistent theory for non-equilibrium processes which may be approximated by quasicontinuum method. These are, of course, the first steps in this direction and much further work is required towards construction of a sound model for nonequilibrium simulations.

\section{References}

Abraham F F, Dchneider D, Land B, Lifka D, Sovira J, Gerner J and Rosenkrantz M 1997 J. Mech. \& Phys. Solids 451461 Belytschko T and Smolinski P 1985 Comput. Meth. Appl. Mech. \& Eng. 49281

Belytschko T and Lu Y Y 1993 Comput. Meth. Appl. Mech. \& Eng. 108353

Belytschko T, Yen H-J and Mullen R 1979 Comput. Meth. Appl. Mech. \& Eng. 17/18 259

Ercolessi F and Adams J 1993 Europhys. Lett. 26583

Goldstein H 1980 Classical mechanics (Reading, Mass: Addison-Wesley)

Hughes T J R 1987 The finite element method (Englewood Cliffs, New Jersey: Prentice-Hall)

King A H and Smith D A 1980 Acta Crystallogr. A36 335 
Shen Z, Wagoner R H and Clark W A T 1988 Acta Metall. 36 3231

Shenoy V B 1998 Quasicontinuum models of atomic-scale mechanics, $\mathrm{Ph} \mathrm{D}$ thesis, Brown University, Providence, RI, USA

Shenoy V B, Miller R M, Tadmor E B, Phillips R and Ortiz M 1998 Phys. Rev. Lett. 80742
Shenoy V B, Miller R M, Tadmor E B, Rodney D, Phillips R and Ortiz M 1999 J. Mech. \& Phys. Solids 47611

Tadmor E B 1996 The quasicontinuum method, Ph D thesis, Brown University, Providence, RI, USA

Tadmor E B, Ortiz N and Phillips R 1996 Philos. Mag. A73 1529 